\title{
Constitutive activation of mTOR signaling pathway in post-transplant lymphoproliferative disorders
}

\author{
Mouna El-Salem ${ }^{1}$, Puthiyaveettil N Raghunath ${ }^{1}$, Michal Marzec ${ }^{1}$, Pawel Wlodarski ${ }^{1}$, \\ Donald Tsai ${ }^{2}$, Eric $\mathrm{Hsi}^{3}$ and Mariusz A Wasik ${ }^{1}$ \\ ${ }^{1}$ Department of Pathology and Laboratory Medicine, University of Pennsylvania Medical Center, \\ Philadelphia, PA, USA; ${ }^{2}$ Department of Medicine, University of Pennsylvania Medical Center, Philadelphia, \\ PA, USA and ${ }^{3}$ Department of Pathology, Cleveland Clinic Foundation, Cleveland, OH, USA
}

\begin{abstract}
We examined activation of the mTOR signaling pathway in situ in the primary, normal reactive and patientderived post-transplant lymphoproliferative disorder (PTLD) tissue samples. We accomplished this analysis by immunohistochemistry on formalin-fixed, paraffin-embedded specimens using a set of highly specific antibodies that permitted us to determine phosphorylation status of the key serines in the mTOR target proteins. Our results demonstrate that the mTOR signaling pathway is activated in reactive tissue in a highly distinct fashion with positive, typically enlarged cells being present primarily in the germinal center and, to a lesser degree, in interfollicular areas with mantle zone being conspicuously negative. We could demonstrate mTOR activation in the lesional cells in the entire spectrum of PTLD subtypes, regardless of their Epstein-Barr virus genome expression status. These data demonstrate the ubiquitous activation of the mTOR signaling pathway in PTLD and indicate that mTOR inhibitors may be effective in treatment and, notably, prevention of PTLDs given their immunosuppressive properties. Furthermore, our results define potential biomarkers of the therapeutic response. Because the constitutive mTOR activation has also been identified in cells isolated from other hematologic malignancies, the ability to examine the in vivo mTOR signaling may have implications reaching beyond the PTLD field.
\end{abstract}

Laboratory Investigation (2007) 87, 29-39. doi:10.1038/labinvest.3700494; published online 30 October 2006

Keywords: 4E-binding protein 1; eukaryotic initiation factor 4G; mammalian target of rapamycin; post-transplant lymphoproliferative disorder; rapamycin; S6 ribosomal protein

Post-transplant lymphoproliferative disorders (PTLDs) represent a serious complication of standard immunosuppressive therapy and are sometimes lethal despite therapeutic interventions. ${ }^{1}$ PTLDs comprise a rather heterogeneous group of aberrant lymphocytic expansions ranging histologically from a hyperplasia to an overt lymphoma, usually of B-lymphocyte origin. ${ }^{1,2}$ According to the WHO classification ${ }^{1}$ PTLDs can be divided into at least four broad, yet distinct categories: (1) early lesions, such as plasmacytic hyperplasias and infectious mononucleosis-like lesions, which are

Correspondence: Dr MA Wasik, MD, Department of Pathology and Laboratory Medicine, University of Pennsylvania Medical Center, 3400 Spruce Street, 7.106 Founders Pavilion, Philadelphia, PA 19104, USA.

E-mail: wasik@mail.med.upenn.edu

Received 6 June 2006; revised and accepted 28 September 2006; published online 30 October 2006 usually polyclonal, (2) polymorphic PTLDs, which almost always show clonal immunoglobulin gene rearrangement in PCR assay, (3) monomorphic PTLDs, which comprise overt B-cell neoplasms such as diffuse large B-cell lymphoma, Burkitt/ Burkitt-like lymphoma, plasma cell myeloma, and T-cell lymphomas, all of which virtually always are monoclonal and frequently display structural genetic abnormalities, and (4) Hodgkin/Hodgkin-like PTLD. The pathogenesis of PTLDs is not fully understood. Epstein-Barr virus (EBV) has long been implicated in the development of PTLD in the majority of cases ${ }^{3,4}$ with some of the EBV-encoded proteins displaying cell-transforming properties. ${ }^{3,4}$ Whereas most of the EBV-containing PTLDs express broad spectrum of the EBV-encoded genes (type III latency), rarely a more limited expression of the EBV genome (type I latency) can be encountered. ${ }^{5}$

Mammalian target of rapamycin (mTOR) is a ubiquitously expressed and highly conserved ser- 
ine/threonine kinase that affects a number of key cell functions including protein synthesis and cell proliferation. mTOR acts by directly activating p70S6 kinase (p70S6K) (also known as S6K1) and inhibiting $4 \mathrm{E}$ binding protein 1 (4E-BP1), also known as PHAS-1 ${ }^{6-14}$ p70S6K is a serine/threonine kinase that phosphorylates a S6 protein of the 40S ribosomal subunit (S6rp, also known as S6 ribosomal protein) at several sites including serines 235 and $236 .{ }^{15-17}$ In turn, 4E-BP1 is a translational repressor that negatively regulates eukaryotic initiation factor $4 \mathrm{E} / 4 \mathrm{G}$ (eIF-4E/G) complex by modulating phosphorylation of the involved proteins. ${ }^{18}$ The exact mechanisms leading to mTOR activation are still under investigation, but certain nutrients and growth factors are believed to act as indirect activators of mTOR ${ }^{19-22}$ by suppressing activity of tuberous sclerosis complex TSC1 and TSC2 proteins that, in turn, inhibit activity of mTOR through inactivating the $G$ protein Rheb. ${ }^{23}$ mTOR activity can be inhibited by rapamycin and related small molecule compounds. Rapamycin and its derivatives are currently used clinically as immunosuppressive $\operatorname{drugs}^{24,25}$ and evaluated as potential therapeutic agents in several types of cancer. $^{26-29}$

Our previous studies ${ }^{8,24,30}$ suggested that mTOR signaling may play a role in the pathogenesis of PTLDs. Specifically, we have shown that EBV-transformed cells are sensitive to mTOR inhibitors both in vitro and in vivo using a xenotransplant model. Furthermore, we have determined that mTOR activation can be detected in various types of transformed B cells regardless of their status of EBV genome expression and activation of Akt, the serine/threonine kinase implicated previously in mTOR regulation. ${ }^{30}$ However, these studies were performed on cultured cell lines, some of which being developed by in vitro infection with EBV of peripheral blood mononuclear cells obtained from normal healthy individuals $^{24,30}$ and only one being established directly from a PTLD tissue. ${ }^{8}$ Therefore, these cell lines represented rather imperfect models of PTLD.

In this study, we examined activation of the TOR signaling pathway in PTLD in vivo using the primary, patient-derived tissue samples representing various types of PTLD. We accomplished this task by performing immunohistochemistry on the formalin-fixed, paraffin-embedded PTLD tissues with a set of highly specific antibodies that permit to determine phosphorylation status of the key serines or threonines on the mTOR target proteins. Our results demonstrate that mTOR signaling pathway is activated in the entire spectrum of PTLD subtypes regardless of their EBV genome expression status. Consequently, these data provide evidence in support of the notion that mTOR inhibitors may be effective in treatment and, notably, prevention of PTLDs.

\section{Materials and methods}

\section{Cell Lines}

The EBV-negative cell lines, LY18 and Val, were derived from diffuse large B-cell lymphoma and Ramos from Burkitt lymphoma. The EBV-positive, latency type I cell line Raji was derived from Burkitt lymphoma and the EBV-positive, latency type III lymphoblastoid B-cell lines (LCL), MM, HH, were established in our laboratory by the EBV-mediated immortalization of peripheral blood B lymphocytes as described. ${ }^{24}$ Lymphoblastoid cell lines obtained by this method has been proven to induce human B-cell lymphoma in mice roughly corresponding to the monomorphic type of PTLD in humans. ${ }^{24}$

\section{PTLD Tissue Samples}

We examined tissue samples from a total of 38 patient samples diagnosed with PTLD following solid organ transplant. The cases were identified in the files of the Department of Pathology and Laboratory Medicine at the Hospital of the University of Pennsylvania, Children Hospital of Philadelphia and Cleveland Clinic Medical Center. The corresponding formalin- and/or B5-fixed paraffinembedded tissue samples were retrieved. The samples represented small diagnostic and large excisional biopsies of different solid organs. All cases were classified as PTLDs based on clinical history, morphology, immunohistochemistry, flow cytometry, and, in most cases, EBV gene expression. The cases studied were comprised of six early PTLDs, five polymorphic B-cell PTLD, and 27 monomorphic PTLD. Of the 38 evaluated PTLDs, 33 expressed the EBV genome as determined by in situ hybridization for EBER-1 RNA using the FITClabeled oligo probe for EBER-1 (as described in the Materials and methods). Of the 20 EBER-1-positive cases that were tested for the late membrane protein 1 (LMP-1), 18 were also positive for (LMP-1) by immunohistochemistry with the appropriate antibody (DakoCytometion, Carpinteria, CA, USA).

\section{Western Blotting}

The cells were washed briefly in PBS, centrifuged and lysed in RIPA buffer supplemented with $0.5 \mathrm{mM}$ PMSF, phosphatase inhibitor cocktails I and II (Sigma, St Louis, MO, USA) and protease inhibitor cocktail (Roche, Mannheim, Germany) according to the manufacturer's specifications. For normalization of the gel loading, the protein extracts were assayed with Lowry method (Bio-Rad Dc protein assay, Hercules, CA, USA). Typically, $20 \mu \mathrm{g}$ micrograms of the protein per lane were loaded. To examine protein expression and phosphorylation, the membranes were incubated with antibodies specific for phospho (p)-p70S6K (Thr389), total p70S6K, p-S6rp 
(Ser235/236), total S6rp, p-4E-BP1 (Thr37/46), p-4EBP1 (Ser65), total 4E-BP1, p-eIF-4G (Ser1108), total p-eIF-4G, p-TSC2 (Thr 1462), total TSC2, p-Akt (Thr308) and total Akt (all antibodies were from Cell Signaling, Danvers, MA, USA). Next, the membranes were incubated with the appropriate secondary, peroxidase-conjugated antibodies. The blots were developed using the Lumingen PS-3 detection reagent from Amersham Biosciences, Pittsburgh, PA, USA.

\section{Densitometry}

Intensity of the signal was measured using scanner HP ScanJet3500c and ImageJ 1.32j software developed at the National Institute of Health, Bethesda, MD, USA.

\section{mTOR Inhibitor}

Rapamycin was purchased from Cell Signaling. Rapamycin was reconstituted in DMSO to the suitable stock concentration and diluted shortly before the experiments to the final concentration of $10 \mathrm{nM}$.

\section{Immunohistochemistry}

Immunohistochemistry was carried out using the following antibodies: p-4E-BP1 (Ser65), p-eIF-4G (Ser1108), and p-S6rp (Ser235/236) (all from Cell Signaling). For the p-eIF-4G and p-S6rp, a streptavidin horseradish immunoperoxidase method $^{31}$ was used with some modifications. Sections $(5 \mu \mathrm{m})$ from formalin-fixed, paraffin-embedded tissue specimens were deparaffinized in xylene and rehydrated in graded alcohol. After deparaffinization, heat-induced antigen retrieval by boiling slides in $10 \mu \mathrm{mol}$ citrate buffer $\mathrm{pH} 6$ (Lab vision, Fremont, CA, USA) for $20 \mathrm{~min}$ was employed. The sections were blocked for $10 \mathrm{~min}$ with peroxidase blocking system from DakoCytometion. Sequential incubations included the following: $2 \%$ normal goat serum (DakoCytometion) for $20 \mathrm{~min}$; affinity-purified rabbit polyclonal primary antibodies at optimized dilution; secondary biotinylated anti-rabbit IgG (Vector Laboratories Inc., Burlingame, CA, USA) for $30 \mathrm{~min}$; the streptavidinbiotinylated horseradish peroxidase complex reagent (DakoCytometion) for $30 \mathrm{~min}$ at room temperature. p-eIF-4G primary antibody was incubated at room temperature for $60 \mathrm{~min}$ while the other primary antibodies were incubated at $4^{\circ} \mathrm{C}$ overnight. After each incubation, the slides were washed three times in buffer for $3 \mathrm{~min}$. Sections were then exposed to the chromogen DABplus from DakoCytometion, for $5 \mathrm{~min}$ and were counterstained in hematoxylin, dehydrated, cleared and mounted. For p-4E-BP1 antibody, adequate staining was obtained using the protocol in DakoCytometion's catalyzed signal amplification system kit that included a step of blocking any potential endogeneous biotin. For immunocytochemistry, Raji cell line was exponentially grown, collected, and embedded in paraffin after formalin fixation. The staining was performed using the same protocol as for the PTLD patient tissue samples.

\section{Double Staining for mTOR Effectors and EBER-1}

In selected cases, we have combined in situ hybridization for the EBV-encoded EBER-1 RNA with immunohistochemical staining for p-S6rp. First in situ hybridization was carried out with the FITC-labeled oligo probe for EBER-1 synthesized at the University of Pennsylvania nucleic acid facility. After deparaffinization and hydration, the section is digested with pepsin (Biomed A Corp., Foster City, CA, USA) at $37^{\circ} \mathrm{C}$ for $15 \mathrm{~min}$, followed by blocking with peroxidase for $10 \mathrm{~min}$ at room temperature. Tissue sections were covered with $15 \mu$ l of EBER-1FITC probe/hybridization mixture and covered with glass coverslips. Denaturation was performed at $98^{\circ} \mathrm{C}$ for $6 \mathrm{~min}$ and then immediately hybridizing the slides at $37^{\circ} \mathrm{C}$ for $60 \mathrm{~min}$. After hybridization, the coverslips were removed and the slides were washed in post-hybridization wash buffer for three times. Hybridized DNA probe was visualized with anti-FITC-HRP conjugate (DakoCytometion) diluted 1:40 in Tris-buffer. Sections were covered with FITC-HRP for $60 \mathrm{~min}$ at room temperature. After the incubation, the slides were washed in buffer. The color was developed by exposing tissues to the DAB reagent for $5 \mathrm{~min}$ at room temperature. Sections were washed with the buffer in preparation to be stained for the p-S6rp. Before the staining, antigens were unmasked by boiling in $10 \mathrm{mM}$ citrate buffer $\mathrm{pH} 6$ for $15 \mathrm{~min}$. To visualize the p-S6rp immunostaining, the slides were incubated with Vector-SG substrate (Vector Laboratories) for $5 \mathrm{~min}$ at room temperature. The stained sections were dehydrated and cover-slipped. Double-stained cells showed a mixture of brown (EBER) and bluish-gray (S6rp) tone.

\section{Interpretation of Immunohistochemical Stains}

All stained slides were examined independently by two pathologists with $100 \%$ concordance. The samples were designated as positive or negative with the staining ranging from $20 \%$ to more than $95 \%$ of the lesional cells considered positive. When applicable, interpretation of the staining took into consideration the uneven impact of fixation of large specimens on protein phosphorylation state ${ }^{32}$ (please see Discussion for more details). Nonstained lymphocytes and some other non-lymphoid cells within the PTLD lesions served as internal negative control. Reading was based on the architecture and cellular features by correlating with $\mathrm{H} \& \mathrm{E}$, diagnostic 
immunohistochemical stains (T- and B-cell stains such as CD3, CD4, CD8, CD79a, and CD20), and, occasionally, double staining for the mTOR signaling- and EBV-related molecules as described above. In some cases, Ki 67 stain was examined to correlate the results with the PTLD proliferative index.

\section{Results}

\section{Detection of mTOR Target Proteins with the Phospho-Serine/Threonine-Specific Antibodies}

To confirm activation of the mTOR signaling pathway in the cultured PTLD-type cells and to evaluate

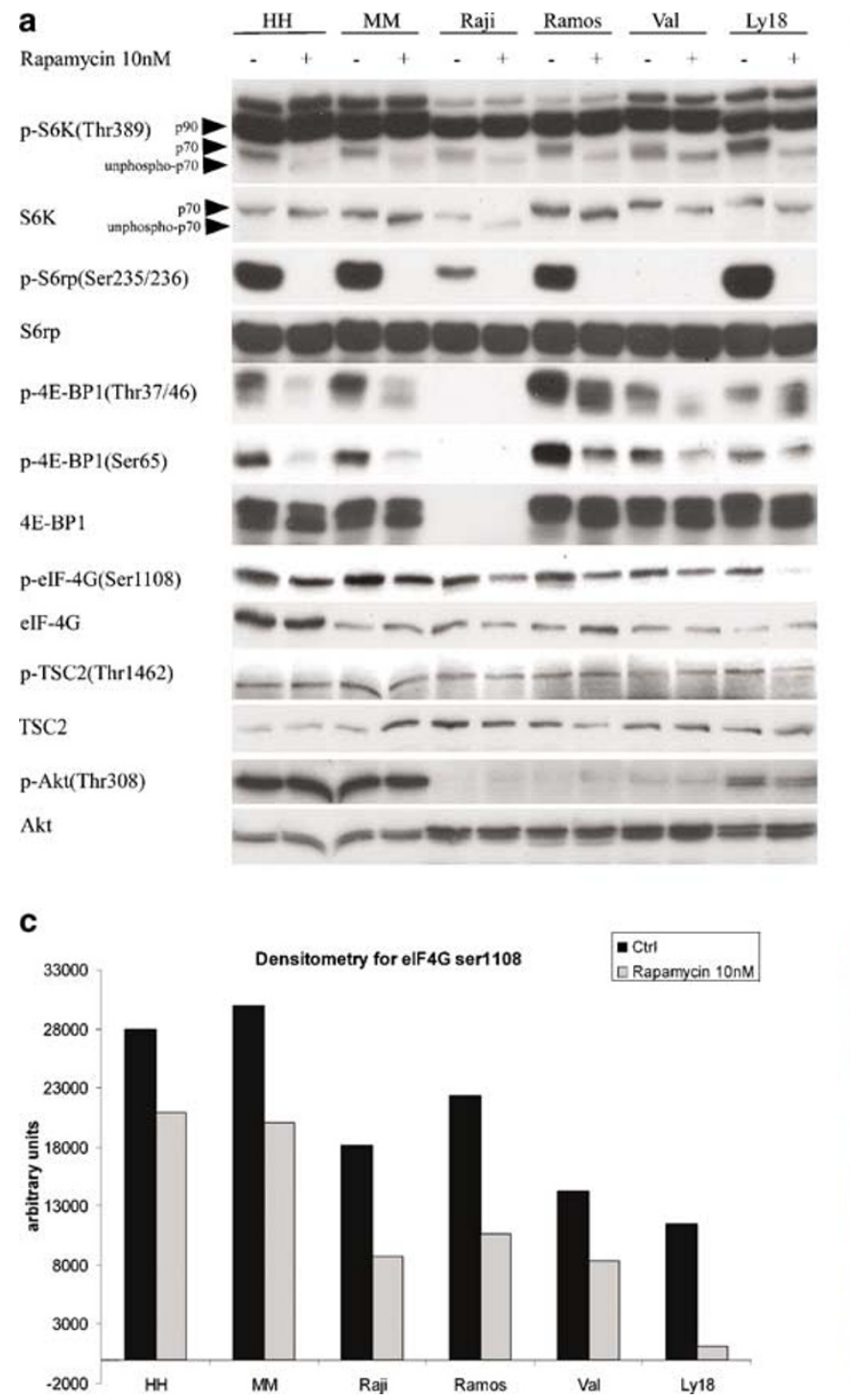

the quality of the available phospho-specific antibodies, we examined a set of transformed Blymphocyte cell lines that varied in their expression of the EBV genome using antibodies against direct and indirect mTOR targets phosphorylated at the key serines and/or threonines. As shown in Figure 1a, we could detect expression of the key mTOR target proteins p70S6K, S6rp, 4E-BP1, and eIF-4G in the phosphorylated (activated) form in all six cell lines examined, regardless of their EBV expression status. Whereas most antibodies generated a single band suggesting their possible suitability for immunohistochemistry (Figure 1a and b), the antibody against phospho-threonine 389 on p70S6K (Figure

\section{b}
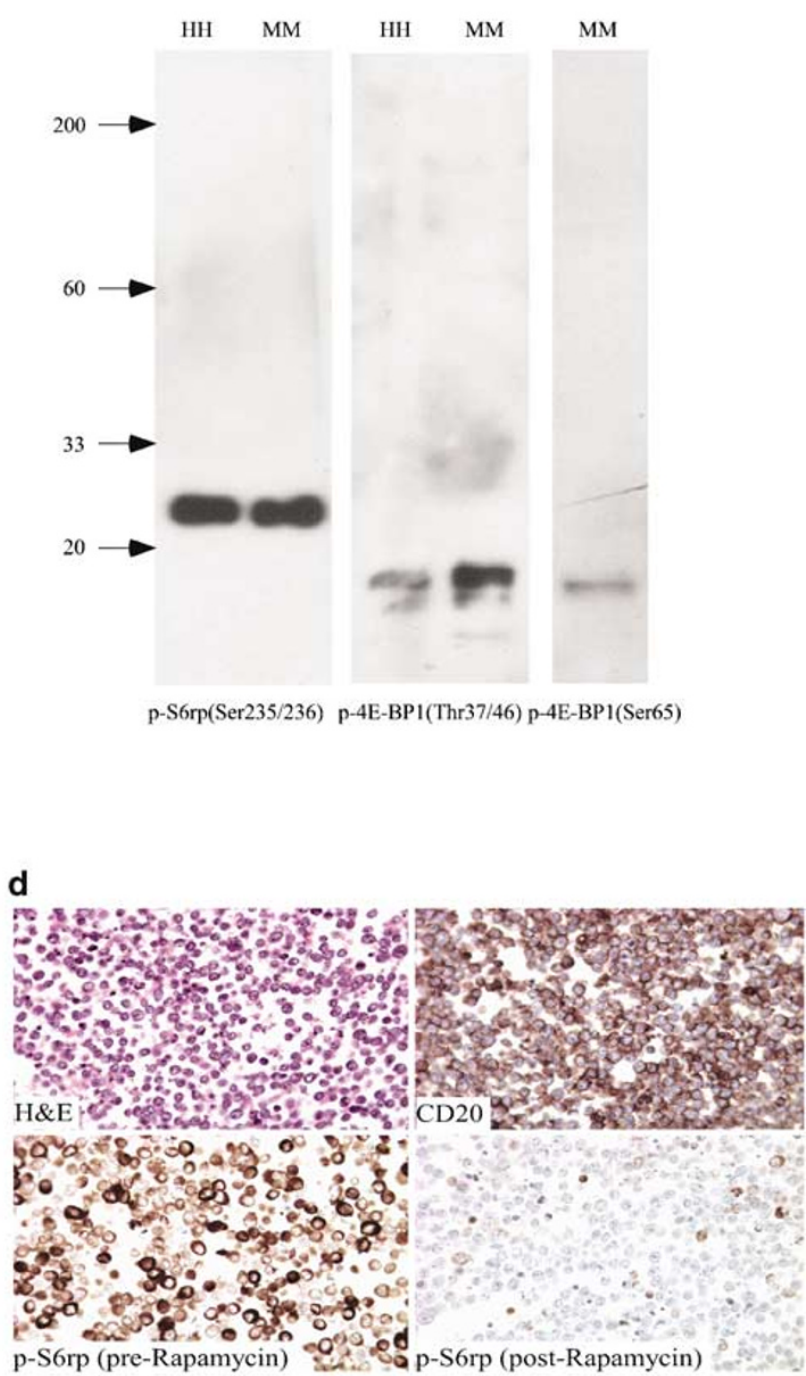

Figure 1 Detection of activated mTOR signaling pathway in cultured PTLD-like B cells. (a) Transformed B-lymphocyte cell lines that were either EBV-positive (HH, MM, and Raji) or negative (Ramos, Val, and Ly18) were incubated for $1 \mathrm{~h}$ in medium or $10 \mathrm{nM}$ of rapamycin and analyzed by Western blotting for activation of mTOR targets using the depicted phospho (p)-specific antibodies. Equal loading of proteins was confirmed by blotting with antibodies against total proteins. (b) The entire Western blots with the depicted key phosphospecific antibodies. The antibodies generated a single band with the protein lysates derived from the EBV-transformed B-lymphocyte cell lines (HH and $\mathrm{MM}$ ), indicating their possible suitability for immunohistochemistry. (c) Densitometric analysis of changes in elF-4G (Ser1108) phosphorylation after cell treatment with rapamycin. (d) Immunocytochemical stains for phospho-S6rp (Ser235/236) was performed on Raji cell line before (lower left) and after (lower right) cell treatment with $10 \mathrm{nM}$ of rapamycin. Upper left: H\&E stain, upper right: stain with an anti-CD20 antibody. All images represent the $\times 400$ magnification. 
1a) detected two additional bands with the most dominant one corresponding to the $90 \mathrm{kDa}$ S6K isoform (p90S6K or RSK), which also expresses the threonine. ${ }^{33}$

To determine if the detected bands indeed correspond to the moieties phosphorylated by mTOR, the cell lines were pretreated with a potent and apparently totally specific mTOR inhibitor, rapamycin. ${ }^{34}$ As can be seen in Figure 1a, the inhibitor used at the low dose of $10 \mathrm{nM}$ was able to essentially completely inhibit phosphorylation of p70S6K, S6rp, and 4E-BP1. Only a partial inhibition of eIF-4G at serine 1108 phosphorylation was also noted (the corresponding densitogram is presented in Figure 1c). As expected, rapamycin had no effect on phosphorylation of the mTOR inhibitor TSC2 and, where present, phosphorylation of Akt. Noteworthy, phosphorylation of p90S6K was also not affected by rapamycin confirming that the p90S6K is not phosphorylated by mTOR. ${ }^{33}$ The crossreactivity of the anti-phospho-Thr389 p70S6K with the strongly expressed phospho-p90S6K indicated that the antibody could not be used for immunohistochemical analysis of mTOR signaling. In contrast, as mentioned above, phosphorylation of S6rp, the target of p70S6K but not of p90S6K, was completely abrogated by rapamycin. This abrogation was additionally visualized by immunocytochemistry using a cell block prepared from one of the cell lines, Raji (Figure 1d).

\section{Activation Pattern of mTOR Signaling Pathway in Tissue Sections of Benign, Reactive Lymphoid Organs}

To examine activation of the mTOR pathway in vivo directly in lymphoid tissues, at the same time, to determine which of the phospho-specific antibodies against mTOR target proteins retain their reactivity in the archival formalin-fixed, paraffin-embedded patient tissues routinely prepared for the diagnostic purposes, we performed an immunohistochemical analysis with the reactive lymph nodes and the above-described, preselected antibodies. This analysis has revealed a highly distinct, pattern of activation of the mTOR pathway (Figure 2). Importantly, all the antibodies examined: antiphospho-S6rp (Figure 2b), -4E-BP1 (Figure 2c), and -eIF-4G (Figure 2d), yielded essentially the same, mutually consistent results in the depicted lymph node and also in the tonsillar tissue (data not shown). Whereas $30-50 \%$ of cells in the follicle germinal center and $10-30 \%$ in the interfollicular area expressed activation of the mTOR pathway, the remaining cells including essentially all cells in the follicle mantle zone were negative. Furthermore, at least a subset of the positive cells displayed large cell morphology suggesting that mTOR pathway is turned on preferentially in the activated cells. This association between cell activation and mTOR signaling was further highlighted by staining for the cell cycle-related MIB1 (Ki-67) antigen (Figure $2 \mathrm{a}$; inset) that also labeled mainly the large-cell subset in the germinal centers and interfollicular area and did not stain mantle zone cells. In addition to the large cells, the identified scattered plasma cells also displayed activation of the mTOR pathway (not shown in detail; please see Figure 5 for plasmacytic hyperplasia), suggesting that high protein synthesis rate might also require activation of mTOR signaling.

\section{Activation of the mTOR Pathway in PTLDs}

To determine activation status of mTOR pathway in PTLD tissues, we evaluated by immunohistochemistry, using the phospho-specific antibodies, a total of 38 cases of PTLD representative of various histological subtypes of the disorder, over threefourths $(76 \%)$ of which contained the EBV genome, as determined by detection of the EBER-1 expression. The representative results of the mTOR signaling pathway activation analysis are depicted in Figures 3-6. We began our analysis with the overt lymphoma type of PTLD in which the malignant cells were easily identifiable by morphology. As can be seen, monomorphic PTLDs of both B-cell and T-cell derivation (Figures 3 and 4, respectively) displayed strong expression of the phospho-form of all the three mTOR targets examined: S6rp, 4E-BP1, and eIF-4G. In most cases, the reactivity was identified in the vast majority, if not all, of the malignant-appearing PTLD cells. The mTOR activation was present in the PTLDs regardless of their EBV-genome expression status.

In contrast to the PTLD with morphology of overt malignancies, in the biologically earlier lesions from the pleomorphic and, in particular, hyperplastic categories, it is often difficult to distinguish the (pre-) malignant PTLD cells from the frequently dominant reactive cell component. As shown in Figure 5, this was not the case in the plasmacytic hyperplasia where we could demonstrate strong staining of the morphologically easily recognizable plasma cells. Similarly distinct staining was also seen in the clonal extramedullary plasmacytoma-like PTLD (data not presented) indicating, together with the above-mentioned findings in the benign lymphoid tissues, a robust activation of the mTOR pathway in plasma cells, both reactive and malignant. To examine activation of the mTOR pathway in the morphologically less clear PTLD lesions, we employed a two-color, double-staining method using the combination of in situ hybridization for the EBVencoded EBER-1 and the phospho(Ser235/236)S6rp-specific antibody (Figure 6). Three general patterns were noted among the positively staining cells. Whereas most of the positive lymphoid cells expressed either phospho-S6rp or EBER-1 alone, a subset of the cells displayed expression of both of these markers. 

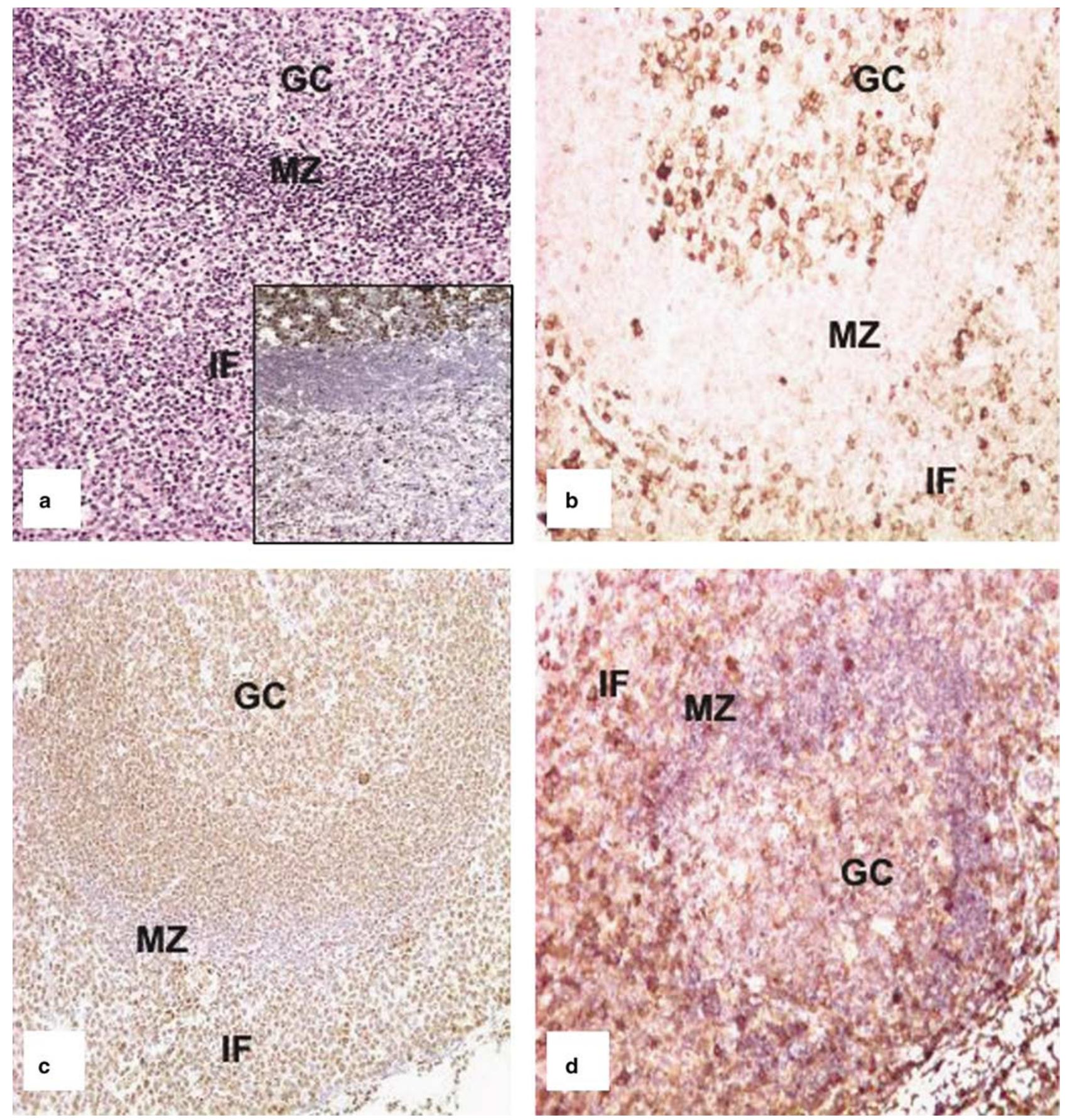

Figure 2 Activation of the mTOR signaling pathway in reactive lymphoid tissue. (a) H\&E stain of a hyperplastic lymph node. GC: germinal center, MZ: mantle zone, IF: interfollicular area. Inset: staining for the cell proliferation-related MIB1 antigen. Immunohistochemical stains to detect expression of S6rp phosphorylated at serine 235/236 (b), 4E-BP1 phosphorylated at serine 65 (c), and eIF-4G phosphorylated at serine 1108 (d). All images represent the $\times 200$ magnification.

\section{Discussion}

The serine/threonine kinase mTOR controls cell growth through several downstream effectors including the 4E-BP1 translational repressor and the p70S6K which, in turn, regulate eIF-4E/G complex and S6rp, respectively. ${ }^{7,18}$ Because p70S6K and 4E$\mathrm{BP} 1$ are involved in protein translation, inhibition of
mTOR function by rapamycin and other structurally related compounds abruptly inhibits the synthesis of critical proteins and profoundly blocks cell cycle progression at the G1 to S phase. ${ }^{27}$ Consequently, mTOR inhibitors are clinically used and actively evaluated as immunosuppressive and anticancer drugs, respectively. Our previous data generated using cultured cell lines strongly suggested that 

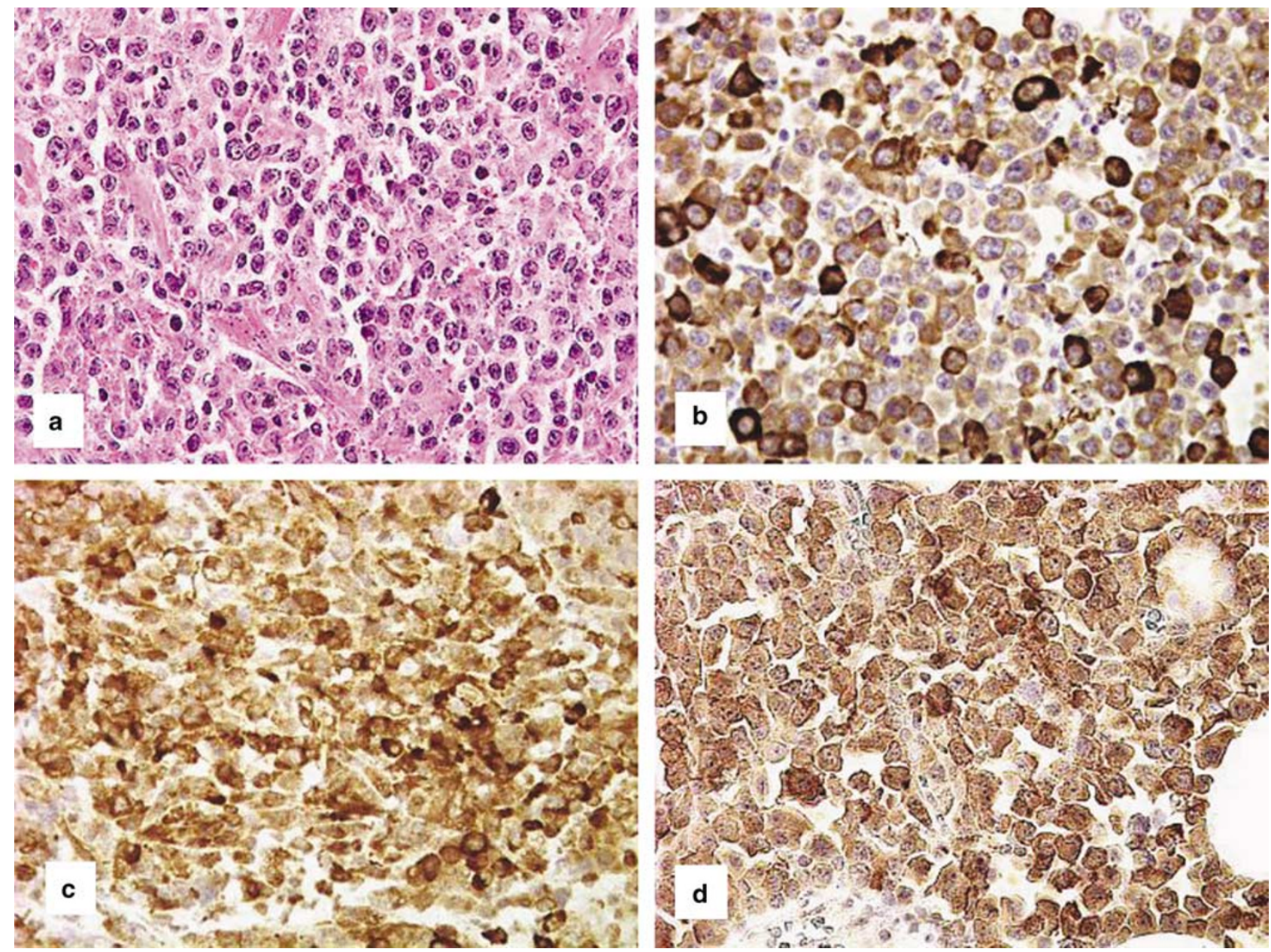

Figure 3 Activation of mTOR pathway in PTLD, monomorphic type, B-cell. (a) H\&E stain of a porta hepatis mass diagnosed as B-cell monomorphic PTLD, comprising sheets of transformed large neoplastic cells. p-S6rp (Ser235/236) immunohistochemical stain shows diffuse cytoplasmic staining in most of the cells with being more intense in the larger cells (b). p-4E-BP1 (Ser65) is also strongly expressed in the neoplastic cells (c) as is the case for p-eIF-4G (Ser1108) (d). All images correspond to the $\times 400$ magnification.

PTLDs may be particularly sensitive to inhibition of the mTOR signaling. ${ }^{8,24,30}$ However, no analysis of mTOR signaling in vivo using tissues derived from PTLDs or normal reactive lymphoid organs has been performed so far. By using immunohistochemical staining with the phospho-specific antibodies, we found that activation of the mTOR signaling pathway can be identified in normal reactive lymphoid tissues and essentially all types of PTLD lesions. Among the normal reactive cells, mTOR pathway activation is particularly prevalent among germinal center B cells and interfollicular T cells with mantle zone B cells showing essentially no staining (Figure $3)$. mTOR activation correlated with large, transformed cell morphology and high proliferative rate. In addition, plasma cells were also positive, suggesting that other cell functional states beside cell proliferation-possibly high rate of protein synthesis-require activation of the mTOR signaling pathway.

In regard to PTLDs, we found that mTOR activation was ubiquitous, if not universal. It spanned all histologic categories examined. It was present in both B- and T-cell PTLDs regardless of their expression status of the EBV genome. The lack of correlation with the EBV status, more specifically mTOR expression in the EBV-negative lesions, is in agreement with activation of mTOR pathway in the normal, reactive tissues, as mentioned above, as well as with our previous finding that both EBVpositive and EBV-negative B-cell lines displayed activation of the pathway. ${ }^{30}$ In fact, we show now that the presence of EBV genome alone is not sufficient to activate the pathway because in a large subset of EBER-1-expressing cells, the indicator of mTOR activity, S6rp, remained unphosphorylated as documented by a double staining to detect EBVencoded EBER-1 and phospho-S6rp (Figure 6).

This study highlights the usefulness of phosphospecific antibodies to study cell signaling in the primary, patient-derived tissues. The antibodies used are directed against the defined phosphorylated serine or threonine moiety, phosphorylation of which is a known step within the pathway under 

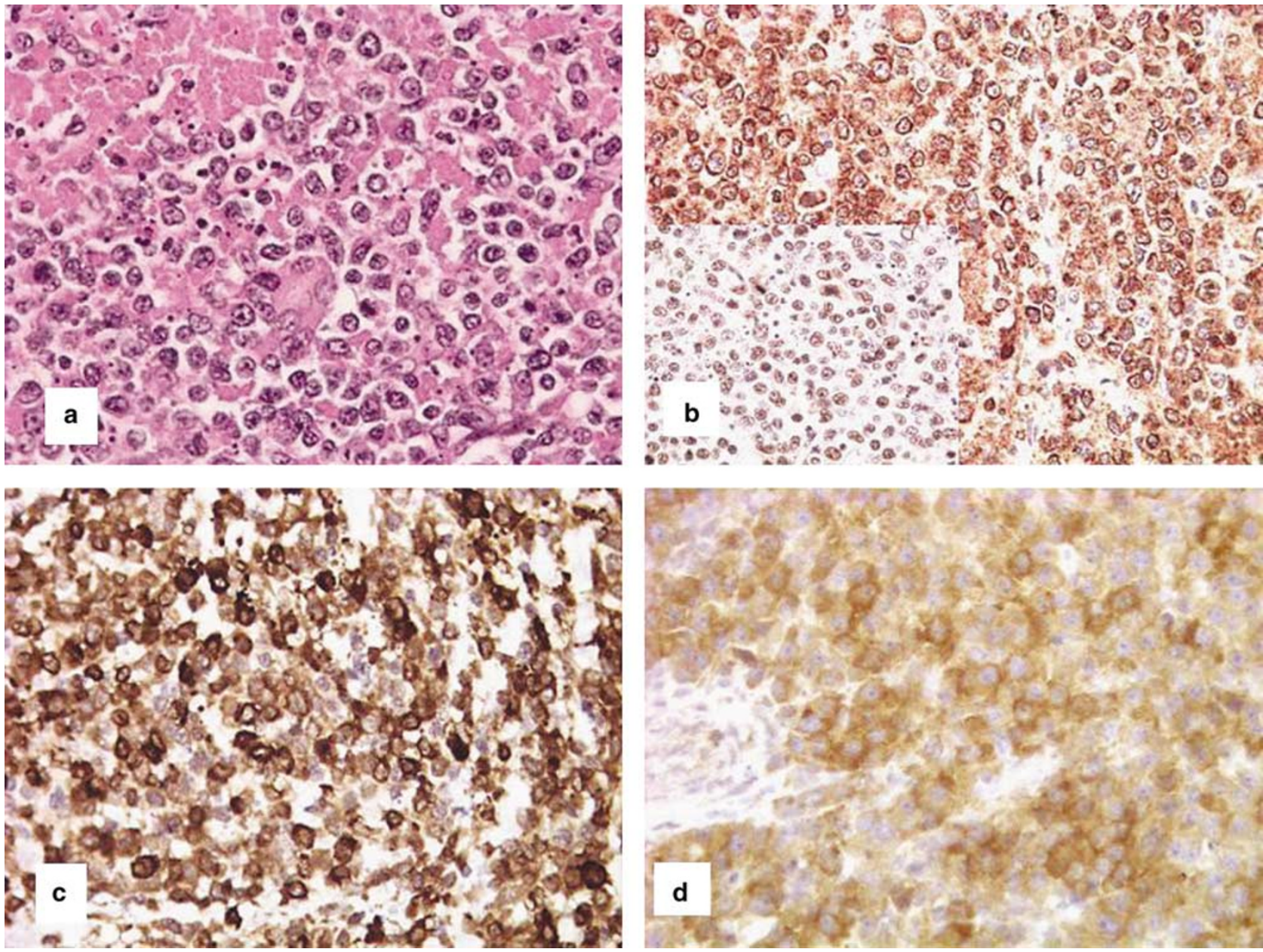

Figure 4 Activation of mTOR pathway in PTLD, monomorphic type, T-cell. Peripheral T-cell lymphoma: a population of large atypical cells with irregular nuclei, ample cytoplasm and increased apoptosis (a: H\&E) expresses CD3 (b) and EBER-1 (inset). The cells express strongly p-S6rp (Ser235/236) (c) and p-eIF-4G (Ser1108) (d). All images represent the $\times 400$ magnification.

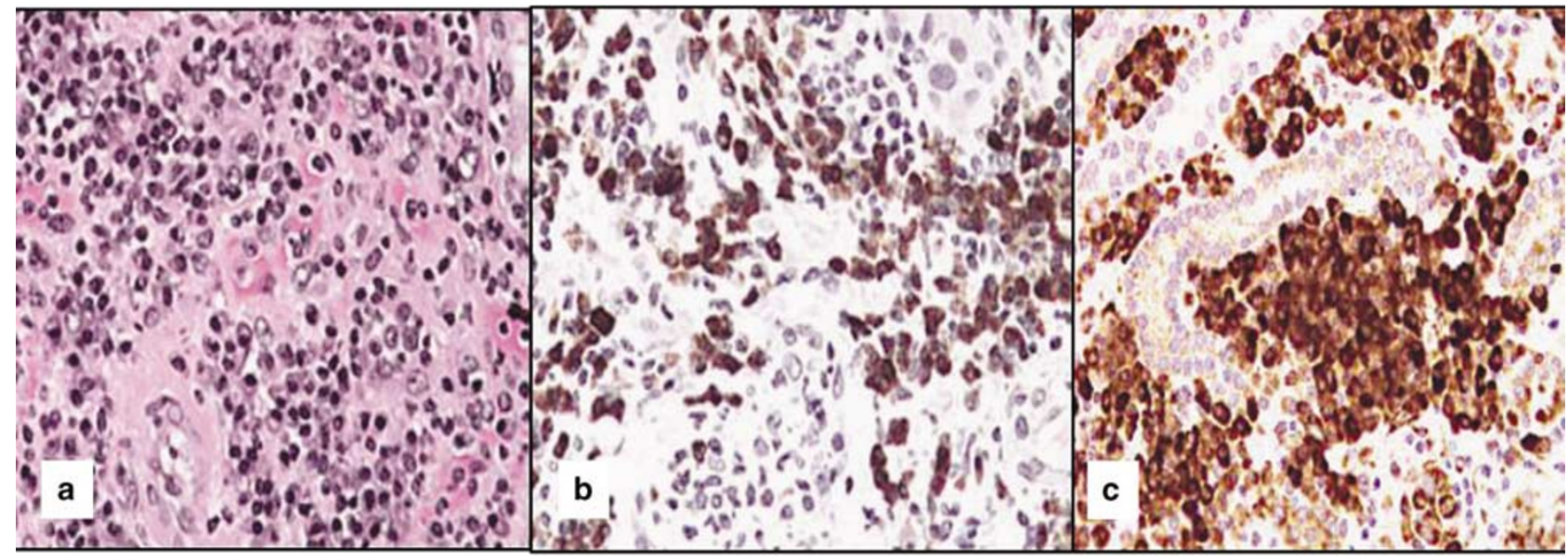

Figure 5 Activation of mTOR pathway in the early PTLD, plasma cell hyperplasia. A section of renal parenchyma diagnosed as involved by plasma cell rich PTLD: aggregates of plasma cells surrounding the renal tubules (a: H\&E) are positive for CD79a (b), p-S6rp (Ser235/ 236) (c). All images are of $\times 400$ magnifications.

study and typically is critical for activation of the evaluated protein. As demonstrated by this and other studies, ${ }^{35,36}$ the phospho-specific antibodies are capable of working in fixed tissues, particularly if antigen retrieval methods are employed to unmask the antigen. This approach is clearly superior to the 

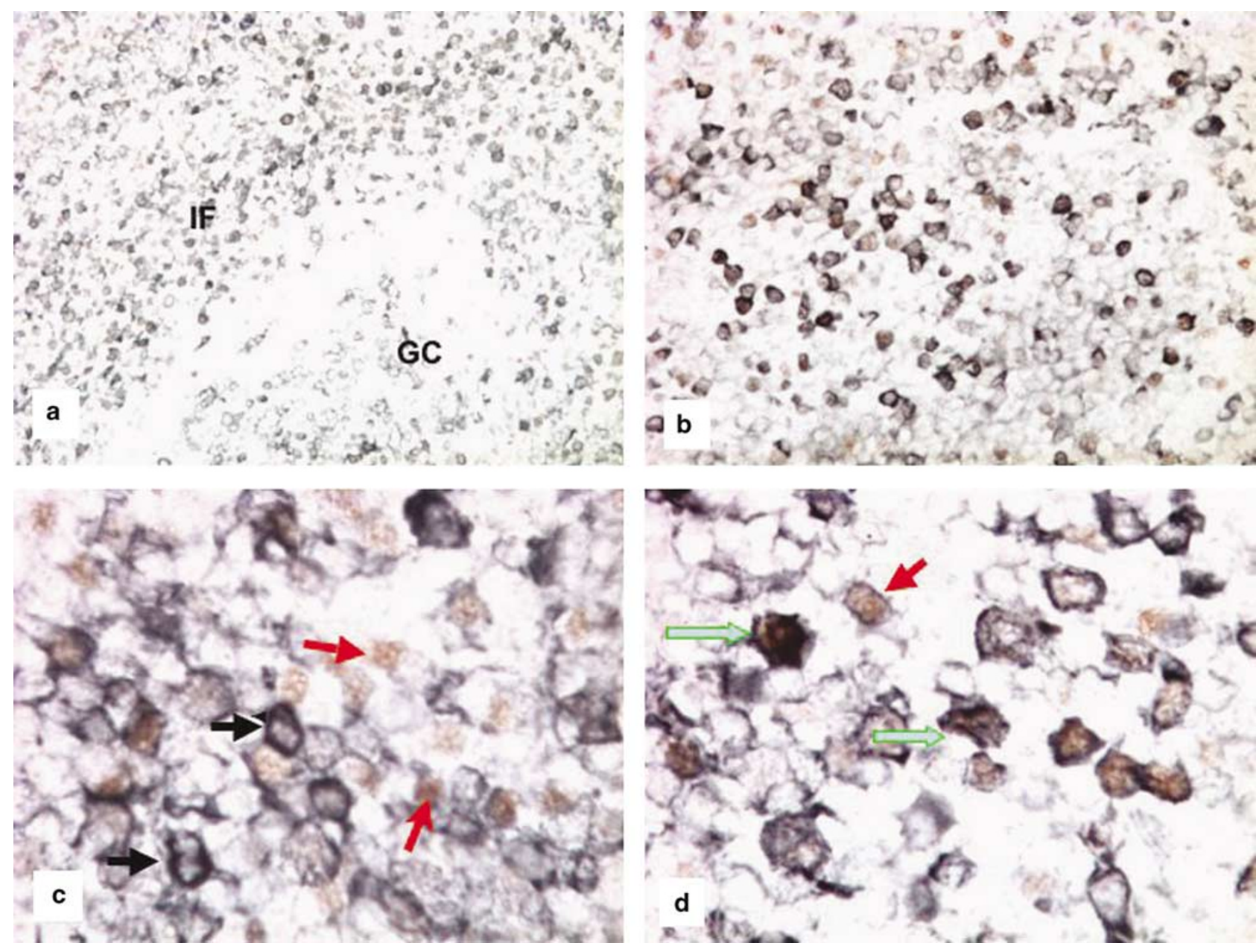

Figure 6 Relationship between EBV expression and mTOR activation in the early stage PTLD. Immunohistochemical double staining for p-S6rp (Ser235/236) and EBER-1 shows three patterns: most of the scattered activated lymphoid cells in the germinal center and interfollicular areas expressed either cytoplasmic p-S6rp (black arrows) or nuclear EBER-1 (red arrows), only a small number of cells showed double expression of EBER-1 and p-S6rp (green arrows), (a) $\times 200$, (b) $\times 400$, and (c) and (d) $\times 1000$ magnification, oil.

conventional Western blotting-based approaches, mainly because PTLDs typically contain various proportions of reactive cells in addition to malignant lymphocytes. Therefore, the result of the Western blotting may not be informative in regard to PTLD cell activation due to the lack of morphologic correlation to exclude normal reactive cells. In addition, the relatively long time required for cell isolation and protein purification to perform the blotting may lead to a decrease, or even loss, of the phosphorylation. By the same token, we cannot totally exclude that the occasional lack of staining observed by us did not represent a false negative result caused by suboptimal formalin fixation of the examined tissue. Because the fixation process relays on the time-dependent penetration of the formalin fixative, the superficial layers of large specimens typically show preservation of the phosphorylation state, whereas tissue deep in the core tends to lose phosphorylation by the time that the fixative has permeated. For this reason, we, as well as others using different experimental systems, ${ }^{35,36}$ limited analysis of immunoreactivity to the well fixed areas of the PTLD tissue represented in outer portion of larger excisional biopsies avoiding at the same time the surface of the specimens which in general are prone to generate false positive staining. The admixed nonstaining lymphocytes and some other non-malignant cell types served as an internal negative control.

Selection of the proper mTOR targets as biomarkers of activation of the mTOR signaling pathway is of critical importance. In our study, evaluation of both p-4E-BP1 and p-S6rp expression was highly informative showing a good correlation between the two staining patterns. However, evaluation of p-eIF $4 \mathrm{G}$ may be less reliable given the apparent involvement of another, mTOR-independent serine kinase that also targets eIF-4G at the same sites as mTOR (Figure 1). Immunohistochemical evaluation of p70S6K phosphorylation, although in principle very attractive, in practice would be quite misleading because of the structural similarity to the p90S6K (also known as RSK) that is not phosphorylated by 
mTOR (Figure 1). Our data indicate that evaluation of phosphorylation of either 4E-BP1 or S6rp should be very informative in regard to activation of the mTOR pathway. Noteworthy in this context, function of $4 \mathrm{E}-\mathrm{BP} 1$ seems more critical because phosphorylation of p70S6K was found to be dispensable for the mTOR-mediated oncogenicity in the in vitro system ${ }^{32}$ with the caveat that similar finding has not been made so far in the primary malignant cells.

Our finding that the native PTLD cells express activation of the mTOR pathway in vivo in essentially all cases regardless of the histologic type and the EBV status has important clinical implications. This observation substantiates and expands the notion based on our previous findings made with cultured B-cell lines ${ }^{8,24,30}$ that PTLDs should be responsive to inhibition of the mTOR signaling. PTLDs seem to be particularly attractive therapeutic targets for mTOR inhibitors for at least two reasons. First, given that mTOR inhibitors were originally developed as immunosuppressive agents, malignancies derived from lymphocytes should be particularly sensitive to such drugs. Indeed, $\mathrm{IC}_{50}$ to inhibit in vitro cell proliferation for a large spectrum of transformed B lymphocytes is well below $10 \mathrm{nM}^{8,24,30}$ Perhaps more importantly and quite uniquely, mTOR inhibitors should act in the setting of PTLD as dual-function drugs by suppressing growth of PTLD cells and, at the same time, preventing immune rejection of the transplanted organ. These combined properties favorably distinguish mTOR inhibitors from the standard immunosuppressive drugs from the calcineurin inhibitor family (cyclosporin A and FK506) that display no inhibitory effect on PTLD cells. Consequently, mTOR inhibitors may be effective not only in treatment of the already established PTLD but also may prove beneficial in prevention of the PTLD development. Preliminary therapeutic trials in PTLD using rapamycin either alone ${ }^{37}$ or in combination with an anti-B-cell (CD20) antibody ${ }^{38,39}$ have been undertaken and yielded promising results. It has also been recently demonstrated that inhibition of the mTOR pathway and glycolysis in vitro would synergistically impact the energy metabolism in cancer cells and may serve as an effective therapeutic strategy to kill malignant cells by combining the cytostatic effect of mTOR on downstream phosphorylation and ATP depletion of malignant cells. ${ }^{40}$

In summary, our results not only provide proof-ofprinciple evidence that mTOR inhibitors should be effective in the broad spectrum of PTLDs but also define potential biomarkers of the therapeutic response. Given that constitutive mTOR activation has been identified in cells derived from a large spectrum of hematopoietic and nonhematopoietic malignancies, ${ }^{28,29,41,42}$ the in situ immunohistochemical analysis of mTOR signaling may impact also on research and, possibly, patient management, in other non-PTLD types of neoplastic disorders.

\section{Acknowledgements}

This study was supported in part by the NIH Grants R01-CA96856 and R01-DE-017337.

\section{References}

1 Harris NL, Swerdlow SH, Frizzera G, et al. Posttransplant lymphoproliferative disorders. In: Jaffe E, Harris NL, Stein H, Vardiman J (eds). World Health Organization Classification of Tumours, Tumours of the Hematopoietic and Lymphoid Tissues. IARC Press: Lyon, 2001, pp 264-271.

2 Hsieh WS, Lemas MV, Ambinder RF. The biology of Epstein-Barr virus in post-transplant lymphoproliferative disease. Transpl Infect Dis 1999;1:204-212.

3 Tao J, Wasik MA. Epstein-Barr virus associated polymorphic lymphoproliferative disorders occurring in non-transplant settings. Lab Invest 2001;81: $429-437$.

4 Küppers R. B cells under influence: transformation of B cells by Epstein-Barr virus. Nat Rev Immunol 2003; 3:801-812.

5 Paessler M, Kossev P, Tsai D, et al. Expression of SHP-1 phosphatase indicates post-germinal center cell derivation of B-cell posttransplant lymphoproliferative disorders. Lab Invest 2002;82:599-606.

6 Dufner A, Andjelkovic M, Burgering BM, et al. Protein kinase B localization and activation differentially affect S6 kinase 1 activity and eukaryotic translation initiation factor 4E-binding protein 1 phosphorylation. Mol Cell Biol 1999;19:4525-4534.

7 Tee AR, Blenis J. mTOR, translational control and human disease. Semin Cell Dev Biol 2004;16: 29-37.

8 Majewski M, Korecka M, Joergensen J, et al. Immunosuppressive TOR kinase inhibitor everolimus (RAD) suppresses growth of cells derived from posttransplant lymphoproliferative disorder at allograft protecting doses. Transplantation 2003;75:1710-1717.

9 Parsons R. Human cancer, PTEN and the PI-3 kinase pathway. Semin Cell Dev Biol 2004;15:171-176.

10 Fang X, Yu SX, Lu Y, et al. Phosphorylation and inactivation of glycogen synthase kinase 3 by protein kinase A. Proc Natl Acad Sci USA 2000;97:1196011965.

11 Sakamoto K, Hirshman MF, Aschenbach WG, et al. Contraction regulation of Akt in rat skeletal muscle. J Biol Chem 2002;277:11910-11917.

12 Roux PP, Ballif BA, Anjum R, et al. Tumor-promoting phorbol esters and activated Ras inactivate the tuberous sclerosis tumor suppressor complex via p90 ribosomal S6 kinase. Proc Natl Acad Sci USA 2004; 101:13489-13494.

13 Tee AR, Anjum R, Blenis J. Inactivation of the tuberous sclerosis complex-1 and -2 gene products occurs by phosphoinositide 3-kinase/Akt-dependent and -independent phosphorylation of tuberin. J Biol Chem 2003;278:37288-37296.

14 Shaw RJ, Bardeesy N, Manning BD, et al. The LKB1 tumor suppressor negatively regulates mTOR signaling. Cancer Cell 2004;6:91-99.

15 Dufner A, Thomas G. Ribosomal S6 kinase signaling and the control of translation. Exp Cell Res 1999; 253:100-109. 
16 Ferrari S, Bandi HR, Hofsteenge J, et al. Mitogenactivated $70 \mathrm{~K}$ S6 kinase. Identification of in vitro 40S ribosomal S6 phosphorylation sites. J Biol Chem 1991;266:22770-22775.

17 Flotow H, Thomas G. Substrate recognition determinants of the mitogen-activated $70 \mathrm{~K}$ S6 kinase from rat liver. J Biol Chem 1992;267:3074-3078.

18 Sarbassov DD, Sabatini DM. Redox regulation of the nutrient-sensitive raptor-mTOR pathway and complex. J Biol Chem 2005;280:39505-39509.

19 Peng T, Golub TR, Sabatini DM. The immunosuppressant rapamycin mimics a starvation-like signal distinct from amino acid and glucose deprivation. Mol Cell Biol 2002;22:5575-5584.

20 Sakata A, Kuwahara K, Ohmura T et al. Involvement of a rapamycin-sensitive pathway in CD40-mediated activation of murine B cells in vitro. Immunology Lett 1999;68:301-309.

21 Takano A, Usui I, Haruta T, et al. Mammalian target of rapamycin pathway regulates insulin signaling via subcellular redistribution of insulin receptor substrate 1 and integrates nutritional signals and metabolic signals of insulin. Mol Cell Biol 2001;21: 5050-5062.

22 Ozes ON, Akca H, Mayo LD, et al. A phosphatidylinositol 3-kinase/akt/mtor pathway mediates and PTEN antagonizes tumor necrosis factor inhibition of insulin signaling through insulin receptor substrate-1. Proc Natl Acad Sci USA 2001;98:4640-4645.

23 Tee AR, Blenis J. mTOR, translational control and human disease. Semin Cell Dev Biol 2005;16:29-37.

24 Majewski M, Korecka M, Kossev P. The immunosuppressive macrolide RAD inhibits growth of human Epstein-Barr virus-transformed B lymphocytes in vitro and in vivo: a potential approach to prevention and treatment of posttransplant lymphoproliferative disorders. Proc Natl Acad Sci USA 2000;97:42854290 .

25 Nepomuceno RR, Balatoni CE, Natkunam Y, et al. Rapamycin inhibits the interleukin 10 signal transduction pathway and the growth of Epstein-Barr virus B-cell lymphomas. Cancer Res 2003;63:4472-4480.

26 Easton JB, Houghton PJ. Therapeutic potential of target of rapamycin inhibitors. Expert Opin Ther Targets 2004;8:551-564.

27 Rowinsky EK. Targeting the molecular target of rapamycin (mTOR). Curr Opin Oncol 2004;16:564-575.

28 Vignot S, Faivre S, Aguirre D, et al. mTOR-targeted therapy of cancer with rapamycin derivatives. Ann Oncol 2005;4:525-537.

29 Yu K, Toral-Barza L, Discafani C, et al. mTOR, a novel target in breast cancer: the effect of CCI-779, an mTOR inhibitor, in preclinical models of breast cancer. Endocr Relat Cancer 2001;8:249-258.

30 Wlodarski P, Kasprzycka M, Liu X, et al. Activation of mammalian target of rapamycin in transformed $B$ lymphocytes is nutrient dependent but independent of Akt, mitogen-activated protein kinase/extracellular signal-regulated kinase, insulin growth factor-I, and serum. Cancer Res 2005;65:7800-7808.

31 Cartun RW, Pedersen CA. An immunocytochemical technique offering increased sensitivity and lowered cost utilizing a streptavidin horseradish peroxidase conjugate. J Histotechnol 1989;12:273-280.

32 Hosoi H, Dilling MB, Liu LN, et al. Studies on the mechanism of resistance to rapamycin in human cancer cells. Mol Pharmacol 1998;54:815-824.

33 Versteeg HH, Sorensen BB, Slofstra SH, et al. VIIa/ Tissue factor interaction results in a tissue factor cytoplasmic domain-independent activation of protein synthesis, p70, and p90 S6 kinase phosphorylation. J Biol Chem 277;30:27065-27072.

34 Bjornsti MA, Houghton PJ. The TOR pathway: a target for cancer therapy. Nat Rev Cancer 2004;4:335-348.

35 Thomas G, Horvath S, Smith BL, et al. Antibody based profiling of the phosphoinositide 3-kinase pathway in clinical prostate cancer. Cancer Res 2004;10:83518356.

36 Mandell JW. Phosphorylation state-specific antibodies applications in investigative and diagnostic pathology. Am J Pathol 2003;163:1687-1698.

37 Jimenez-Rivera C, Avitzur Y, Fecteau AH, et al. Sirolimus for pediatric liver transplant recipients with post-transplant lymphoproliferative disease and hepatoblastoma. Pediatr Transplant 2004;8:243-248.

38 Garcia VD, Bonamigo-Filho JS, Neumann J, et al. Rituximab and rapamycin for posttransplant lymphoproliferative disease treatment: report of three cases. Transplant Proc 2002;34:2993-2995.

39 Garcia VD, Bonamigo Filho JS, Neumann J, et al. Rituximab in association with rapamycin for posttransplant lymphoproliferative disease treatment. Transpl Int 2003;16:202-206.

$40 \mathrm{Xu}$ RH, Pelicano H, Zhang H, et al. Synergistic effect of targeting mTOR by rapamycin and depleting ATP by inhibition of glycolysis in lymphoma and leukemia cells. Leukemia 2005;19:2153-2158.

41 Frost P, Moatomed F, Hoang B, et al. In vivo anti-tumor effects of the mTOR inhibitor, CCI-779, against human multiple myeloma cells in a xenograft model. Blood 2004;104:4181-4187.

42 Panwalkar A, Verstovsek S, Giles FJ. Mammalian target of rapamycin inhibition as therapy for hematologic malignancies. Cancer 2004;100:657-666. 